\title{
FLORA Y FAUNA DE CUATRO BOSQUES DE Polylepis (ROSACEAE) EN LA CORDILLERA DEL VILCANOTA (CUSCO, PERU).
}

Grace P. Servat ${ }^{1}$, Wilfredo Mendoza $C^{2}$. y José Antonio Ochoa C. ${ }^{3}$

\section{Resumen}

Los bosques de Polylepis ("queñual") de la Cordillera del Vilcanota (3600-4500m) son ecosistemas que contienen una fauna y flora única, caracterizada por especialistas de hábitat y altos niveles de endemismo. Desdichadamente, éstos bosques también representan uno de los hábitats más vulnerables de los altos Andes por la fuerte presión antropogénica existente (tala para leña y materiales de construcción además de sobrepastoreo), ya que constituyen el único recurso maderable en esas alturas. Una de las mayores dificultades en la elaboración de estrategias de conservación para este ecosistema, es el gran vacío de información biológica que se tiene sobre estos bosques. El presente estudio contribuye con información básica dirigida al conocimiento de cuatro localidades de estudio nuevas o poco documentadas para la Cordillera del Vilcanota: Sacsamonte, Yanacocha, Pumahuanca y Queuñamonte. Para cada localidad, se provee información referente a plantas angiospermas (144 especies), aves (68 especies), reptiles y anfibios (7 especies), y mamíferos mayores ( 8 especies). Del reporte faunístico, 9 especies de aves, 1 especie de anfibio y 1 especie de mamífero están listadas como especies con alta prioridad para conservación, en situación indeterminada, vulnerable, o rara. Este reporte es parte del "Proyecto Queñual", el cual tiene por principal objetivo contribuir con un mayor conocimiento de los bosques de Polylepis, para proponer medidas adecuadas para su protección en varias regiones de la Cordillera de los Andes del Perú.

Palabras Claves: Altos Andes, conservación, Cusco, queñual, Queuñamonte, fauna, flora, Perú, Polylepis, Pumahuanca, Sacsamonte, Yanacocha, Vilcanota.

\begin{abstract}
Polylepis ("queñual") forests of the Vilcanota Highlands (3600-4500m) contain a unique flora and fauna characterized by habitat specialists and high levels of endemism. Unfortunately, these forests also represent one of the most vulnerable habitats in the high Andes due to the high anthropogenic pressure existent (cutting for firewood and building materials together with overgrazing), because they constitute the only wood resource at those elevations. One of the major difficulties in the elaboration of conservation strategies for this ecosystem are the large gaps of information existing today. The present study contributes basic information aimed at the knowledge and preservation of four new or poorly known localities for the Vilcanota Highlands: Sacsamonte, Yanacocha, Pumahuanca y Queuñamonte. For each locality, information referring to angiosperm plants (144 species), birds (68 species), reptiles and amphibians (7 species), and large mammals ( 8 species) is provided. From the faunistic report, 9 species of birds, 1 amphibian species, and 1 mammal species, are listed as species with high priority for conservation, threatened, vulnerable or rare. This publication is part of "Proyecto Queñual", whose main objective is to contribute to the knowledge of Polylepis forests in order to propose adequate measurements for its protection in the High Andes of Peru.
\end{abstract}

Keywords: conservation, Cusco, High Andes, queñual, Queuñamonte, flora, fauna, Peru, Polylepis, Pumahuanca, Sacsamonte, Yanacocha, Vilcanota.

\section{Introducción}

La Cordillera del Vilcanota, localizada en el departamento del Cusco, es un componente de la cadena oriental de los Andes del Perú, delimitada por los ríos Vilcatona al sur y Yanatile al norte. La ladera de la vertiente del río Vilcanota, está conformada por numerosas quebradas irrigadas por tributarios del mismo río, así como por el deshielo de nevados. En las partes altas de estas quebradas se encuentran formaciones boscosas de Escallonia ("chachacomo"), Myrcianthes ("unca"), Cytharexylum ("murmuscuy"), y Polylepis ("queñual"); las cuales, representan

\footnotetext{
${ }^{1}$ Department of Biology - R223, University of Missouri - St. Louis, 8001 Natural Bridge Rd., St. Louis, MO 63121; s963071@admiral.umsl.edu.

2 Herbario Vargas (CUZ), Facultad de Ciencias Biológicas, Universidad Nacional de San Antonio Abad del Cusco (UNSAAC); wify@eudoramail.com.

${ }^{3}$ Facultad de Ciencias Biológicas, UNSAAC; ochoas@chaski.unsaac.edu.pe
} 
sistemas biológicos únicos caracterizados por tener distribución restringida (Koepcke 1961), por lo que son de gran interés ecológico, sistemático, y biogeográfico. Sin embargo, y a pesar de su importancia biológica, estos bosques, representan uno de los hábitats más vulnerables de los altos Andes, por la creciente presión humana debido a factores económicos, sociales, y culturales (Venero y De Macedo 1983; Fjeldsa \& Kessler, 1996).

En la Cordillera del Vilcanota, los fragmentos de bosques de Polylepis, se hallan comprendidos entre los 3600 y $4500 \mathrm{~m}$ de altura, usualmente rodeados de formaciones morrénicas desprendidas de las laderas orientales de los Andes. La naturaleza fragmentada de estos bosques ha sido atribuida a especificidad en condiciones fisiológicas y microclimáticas (Weberbauer, 1945; Troll, 1959, 1968; Koepcke, 1961; Walter y Medina, 1969; Simpson, 1979, 1986; Vuilleumier, 1984), aunque también se ha sugerido que estos fragmentos son relictos de un hábitat ampliamente distribuido durante el Pleistoceno (Ellenberg, 1958a,b; Fjeldsa, 1992; Hensen, 1993; Kessler, 1995) el cual se ha ido reduciendo, principalmente debido a factores antropogénicos (tala y quema de bosques además de sobrepastoreo) (Fjeldsa, 1987, 1992; Kessler, 1995; Fjeldsa \& Kessler, 1996).

Los bosques de Polylepis existentes en la Cordillera del Vilcanota contienen poblaciones de aves endémicas como es el caso de Leptasthenura xenothorax $y$ otras especies amenazadas por reducción en el hábitat como Cinclodes excelsior y Anairetes alpinus, razones por las que esta Cordillera, ha sido propuesta como un área prioritaria para conservación (Fjeldsa, 1987; Collar et al., 1992; Wege \& Long, 1995; Fjeldsa \& Kessler, 1996; Stotz et al., 1996). Sin embargo, y a pesar de esto, en lo que a flora y fauna se refiere, los bosques de Polylepis de la Cordillera del Vilcanota, presentan grandes vacíos de información y distan mucho de estar bien documentados. En general, no existen listados básicos de invertebrados ni vertebrados, con excepción de algunas listas de aves realizadas por Fjeldsa (1987) para las cercanías al Nevado Chaiñapuerto, en el valle de Urubamba (3800$4500 \mathrm{~m}$ ), el Abra Málaga en el camino OllantaytamboQuillabamba (4300m); los listados preliminares de aves de Aucca (1993) para el bosque de Yanacocha en Urubamba, y Alegría (1995) para Sacsamonte en Calca. En cuanto a la flora podemos mencionar los estudios de Galiano (1990) en los bosques de Polylepis de la localidad de Yanacocha, Tupayachi (1990) para la flora de los valles de Huarán y Huayoccari (que incluyen las localidades de Sacsamonte y Yanacocha, respectivamente), Holgado (1995) para la quebrada de Panticalle en la comunidad de Puerto Málaga; Béjar (1996) para la flora de los bosques de Polylepis del Valle Sagrado de los Incas (en las localidades de Yanacocha, Sacsamonte y
Pumahuanca); y Aguilar (1998) para la localidad de Mantanay en Urubamba.

El presente estudio tiene por finalidad proporcionar datos básicos como son, reportar nuevas áreas de estudio en la Cordillera del Vilcanota, documentadas con listas de flora $y$ fauna (vertebrados). Este reporte es parte del "Proyecto Queñual", un estudio a largo plazo, el cual tiene por principal objetivo contribuir con un mayor conocimiento de los bosques de Polylepis, para lo cual se vienen realizando estudios ecológicos, de diversidad y riqueza de especies de flora y fauna (vertebrados e invertebrados), cuyo propósito final será proponer medidas adecuadas para la protección de éstos bosques en varias regiones de la Cordillera de los Andes del Perú.

\section{Lugares de estudio}

Las localidades de estudio se localizan en la cadena glacial del Vilcanota (Figura 1), cuyos nevados, tales como el Pumahuanca, Chicón, y Topsan alcanzan los 4500 y $5000 \mathrm{~m}$ de altura. Los bosques estudiados corresponden principalmente a fragmentos de $P$. besseri.

La localidad de Sacsamonte $\left(13^{\circ} 13^{\prime} \mathrm{S}, 72^{\circ} 2^{\prime} \mathrm{O}\right.$, 4050-4150m), se encuentra en la quebrada de Cancha Cancha, en la localidad de Huarán, Provincia de Calca (Figura 1). El bosque se sitúa en un roquerío a ambos lados de un bofedal, rodeado de cerros entre los que destaca el nevado Pukaorqo. La localidad de Yanacocha $\left(13^{\circ} 16^{\prime} \mathrm{S}, 72^{\circ} 3^{\prime} \mathrm{O}, 3800-4200 \mathrm{~m}\right)$, se localiza en la quebrada del mismo nombre, en la localidad de Huayoccari, provincia de Urubamba (Figura 1). El bosque se encuentra rodeando dos lagunas, la de Yanacocha $(250 \times 105 \mathrm{~m})$, y la de Kellococha (100x120m) (Galiano 1990). La localidad de Pumahuanca $\left(13^{\circ} 12^{\prime} \mathrm{S}, 72^{\circ} 5^{\prime} \mathrm{O}, 4110 \mathrm{~m}\right)$, se sitúa en la quebrada de Pacchac en la provincia de Urubamba (Figura 1). El bosque se encuentra en una microcuenca estrecha en un valle colgante. Finalmente, la localidad de Queuñamonte $\left(13^{\circ} 11^{\prime} \mathrm{S}, 72^{\circ} 13^{\prime} \mathrm{O}, 3812 \mathrm{~m}\right)$, se localiza en la quebrada de Yuracmayo en la localidad de Pallata, provincia de Urubamba (Figura 1). El bosque se encuentra sobre un roquerío, situado en la pendiente Este.

Los bosques de Sacsamonte y Queuñamonte se hallan bajo fuerte presión antropogénica siendo el sobrepastoreo y la tala de árboles por conseguir leña los problemas que más los afectan.

Los bosques de Yanacocha y Pumahuanca tienen menor presión humana, en relación con las otras dos localidades, aunque la quema de bosques para incrementar áreas de cultivo representa un problema potencial. 


\section{Métodos}

Los datos para el presente trabajo fueron obtenidos en un total de 16 días por localidad, distribuidos en las siguientes temporadas de campo: Julio 1996, Julio y Septiembre 1997, Mayo, Julio y Setiembre 1998, meses que corresponden a la estación seca. Los datos de anfibios y reptiles se vienen tomando desde 1994, durante la estación lluviosa, principalmente en las quebradas de Pumahuanca y Yanacocha. Estos muestreos se complementaron durante la estación seca en las temporadas de campo mencionadas anteriormente.

Para el estudio florístico se hicieron cuatro transectos en banda de $2 \times 25 \mathrm{~m}$ cada uno, los cuales fueron ubicados al azar en todo el bosque. En cada transecto se evaluó cada metro cuadrado, identificando cada especie y registrando el número de individuos por especie. Así también se hicieron muestreos fuera de los transectos para completar el inventario de la flora.

Los registros de aves, fueron realizados en dos parcelas (cada una de las cuales cubre un área de 200x200m). En cada parcela se hicieron cuatro transectos en banda de $100 \times 20 \mathrm{~m}$ cada uno, y en cada transecto se instalaron puntos fijos de conteo cada 20m. Se hicieron censos de 6:00-8:00am mediante observaciones y grabaciones. Para cada especie observada se tomaron datos del hábitat, sustrato y estrato en el que se encontraban, así también, se grabaron las vocalizaciones de las aves durante 3 minutos en cada punto de conteo usando una grabadora Sony TCM 5000EV con micrófono direccional Seinheiser. Durante el resto del día se complementó el listado de aves con observaciones oportunísticas en varios puntos del bosque.

Para los anfibios y reptiles se efectuaron muestreos tanto diurnos como nocturnos, para los cuales se realizaron búsquedas y observaciones directas principalmente debajo de piedras y musgo, entre la vegetación, así como en ambientes acuáticos (lagunas y riachuelos). Los muestreos nocturnos se llevaron a cabo con la ayuda de una linterna frontal. Para conocer el rango altitudinal se realizaron muestreos a diferentes alturas a lo largo de las quebradas, incluyendo el interior del bosque de Polylepis, las partes altas (pajonal) y las partes inferiores de la quebrada. Los datos se complementaron mediante la revisión de la colección herpetológica del Gabinete de Zoología de la UNSAAC.

Para el presente estudio se incluye una lista preliminar de mamíferos que corresponden a observaciones esporádicas hechas al azar por lo que resulta incompleta.

\section{Resultados}

A continuación, se presentan los resultados obtenidos durante la estación seca para cada localidad estudiada:
Sacsamonte.- Además de Polylepis besseri, presente en la parte inferior del bosque, en la parte superior se encuentra la especie $P$. subsericans. El sotobosque está constituido por 39 especies de plantas herbáceas y arbustivas (Tabla 1). También abundan las plantas epífitas y los musgos que se hallan adheridos a rocas y árboles. Entre los elementos florísticos más comunes del sotobosque se tiene especies de los géneros: Baccharis, Senecio, Fuchsia, y Arenaria lanuginosa, mientras que Cronquistiantus volquensii, Luzula peruviana, Baccharis sp. 2, y Senecio tenuisagittatus, son elementos exclusivos de este bosque (Tabla 1). En lo que se refiere a las aves, se reportan 43 especies, de las cuales el 53.5\% pertenecen al bosque de Polylepis propiamente dicho (Tabla 2). Sólo en esta localidad se registraron Vultur gryphus, especie considerada en situación vulnerable (Pulido 1991), y Asthenes urubambensis, especie amenazada (Wege \& Long 1995), aunque ésta especie solo ha sido reportada por Alegría (1995) por lo que su presencia permanece por confirmar. No se ha hecho hasta la fecha un listado de anfibios y reptiles para esta localidad. En cuanto a los mamíferos se han encontrado las siguientes especies: Pseudalopex culpaeus (Canidae) ("zorro andino"), Conepatus chinga (Mustelidae) ("zorrillo"), Hippocamelus antisensis (Cervidae) ("taruca") y Lagidium peruanum (Chinchillidae) ("vizcacha").

Yanacocha.- Este bosque contiene una de las mayores concentraciones poblacionales de $P$. besseri y $P$. subsericans, además de tener 47 especies de plantas en el sotobosque (Tabla 1). Al igual que en Sacsamonte, hay abundantes plantas epífitas y musgos. Entre los elementos florísticos comunes en el sotobosque se encuentran especies de los géneros Senecio, Baccharis, y Muehlenbeckia volcanica, mientras que entre los elementos florísticos exclusivos están, Cerastium glomeratum, Hackelia mexicana, Melinia peruviana, Barnadesia horrida, Geranium peruvianum, Hieracium sp., Cytharexylum argutedentatum (Tabla 1). El inventario de aves dio como resultado 60 especies, de las cuales el $50 \%$ son del bosque propiamente dicho (Tabla 2). Del listado general es interesante mencionar a Podiceps occipitalis especie común en esta región cuyas poblaciones al norte de su distribución se están reduciendo (Fjeldsa, 1990). El listado de mamíferos incluye $C$. chinga, $H$. antisiensis, Odocoileus virginianus (Cervidae) ("venado de cola blanca") y $L$. peruanum.

Pumahuanca.- Este bosque está constituido por $P$. besseri, aunque en la parte superior existen pequeños grupos aislados de $P$. subsericans. El sotobosque está conformado por 37 especies de plantas (Tabla 1). Al igual que en localidades anteriores, hay abundantes plantas epífitas y musgos. Entre los elementos florísticos comunes en el sotobosque encontramos Senecio rhizomatosus, Bartsia bartsioides y 
Muehlenbeckia volcanica, siendo elementos florísticos exclusivos: Loricaria macbridei, Aa sp., Passiflora trifoliata, Hesperomeles escalloniifolia, y Ourisia chamaedryfolia. Este es el primer listado de aves para esta localidad y se reportan 40 especies, de las cuales $62.5 \%$ pertenecen al bosque propiamente dicho (Tabla 2). También constituye una nueva localidad para $C$. excelsior, A. alpinus y L. xenothorax. En lo que a mamíferos se refiere, se reportaron las siguientes especies: P. culpaeus, Mustela frenata (Mustelidae) ("katay"), H. antisiensis, Lama glama ("llama") y $L$. pacos ("alpaca") (Camelidae), y L. peruanum.

Queuñamonte.- Al igual que en las localidades anteriores el bosque esta constituido por $P$. besseri en la parte inferior y $P$. subsericans en la superior además de 35 especies de plantas de sotobosque (Tabla 1). En esta localidad existen pocas epífitas y musgos en comparación a las localidades anteriores. Entre los elementos florísticos comunes en el sotobosque se tiene: Ageratina sternbergiana $y$ Muehlenbeckia volcanica, mientras que Mutisia cochabambensis, Galium sp., Gaultheria glomerata, y Hesperomeles heterophylla, son elementos florísticos exclusivos de este bosque (Tabla 1). En este trabajo presentamos el primer listado de aves para esta localidad con 34 especies, de las cuales el 50\% pertenecen al bosque propiamente dicho (Tabla 2). También constituye una nueva localidad de estudio para A. alpinus y L. xenothorax. La lista de mamíferos conprende: P. culpaeus, L. glama, L. pacos y $L$. peruanum.

Los anfibios y reptiles reportados para Yanacocha, Pumahuanca y Queuñamonte se presentan en la Tabla 3.

\section{Discusión}

El reporte florístico indica que en los cuatro bosques se encuentran 76 especies de plantas donde predominan la familia Asteraceae, Scrophulariaceae, y Rosaceae. En un trabajo similar para las localidades de Yanacocha, Sacsamonte y Pumahuanca, Béjar (1996) reportó 109 especies de plantas entre las cuales las más importantes familias fueron Asteraceae, Poaceae, Scrophulareaceae, Caryophyllaceae, Rosaceae, Solanaceae, y Apiaceae (Tabla 1). La diferencia en el número de especies es probablemente debido a que en el estudio citado se trabajó durante la estación lluviosa, mientras que el presente estudio sólo se llevó a cabo durante la estación seca que se caracteriza por la ausencia de Poaceas y otras especies sólo presentes durante la estación lluviosa. Si el presente trabajo es complementado con la información bibliográfica existente, se obtiene 144 especies de plantas para las cuatro localidades estudiadas. El bosque con mayor número de especies vegetales es Pumahuanca (83), seguido de Yanacocha (64), Sacsamonte (57), y Queuñamonte (35).
En lo que a aves se refiere, $L$. xenothorax es una especie común en todas las localidades estudiadas, mientras que $A$. alpinus puede ser observada regularmente en todos los bosques, aunque en números muy bajos (3 parejas/localidad), con excepción de Queuñamonte, lugar para el que sólo existe una observación. Algo similar ocurre con $C$. excelsior (2 parejas/localidad). Yanacocha presenta la mayor diversidad de aves (60 especies), seguido de Sacsamonte (43 especies), Pumahuanca (40 especies), y Queuñamonte (35 especies). Estos números incluyen todas las especies reportadas en diferentes hábitats. Sin embargo, las especies de aves que se encuentran en el bosque propiamente dicho varían entre 17 a 30 especies (Tabla 2). En previos listados para otras localidades de la Cordillera del Vilcanota se han reportado hasta 29 especies de aves para el bosque propiamente dicho (Fjeldsa, 1987). Así mientras que Sacsamonte, Yanacocha y Pumahuanca están dentro del rango total de especies esperado, la localidad de Queuñamonte presenta un bajo número. Entre los factores que podrían estar influyendo en este resultado son: menor número de hábitats acuáticos en comparación con las otras localidades, Asi como la poca humedad del bosque. Además de esto, la presión antropogénica existente, particularmente el sobrepastoreo, el cual es intenso en esta localidad.

Del reporte total de aves, $C$. excelsior, A. alpinus, y $L$. xenothorax están listadas como especies en peligro de extinción, o con alta prioridad para conservación (Pulido, 1991; Collar et al., 1992; Wege \& Long, 1995; Stotz et al., 1996), Leptasthenura yanacencis, A. urubambensis, Oreomanes fraseri y Xenodacnis parina como cercanamente amenazadas (Wege \& Long, 1995), V. gryphus como especie en situación vulnerable y Colaptes rupicola como especie en situación rara (Pulido, 1991).

Para anfibios y reptiles, el presente trabajo constituye el primer estudio realizado para estas localidades. La mayoría de especies reportadas presentan un rango altitudinal amplio, con excepción de Pleurodema marmorata, especie que fue hallada únicamente entre los 4200 y $4400 \mathrm{~m}$ en la localidad de Pumahuanca y Phrynopus sp., especie asociada al interior del bosque de Polylepis en la localidad de Queuñamonte. La localidad de Yanacocha presenta la mayor cantidad de especies de anfibios y reptiles (5 especies), seguida de Pumahuanca (4 especies) y Queuñamonte (4). Aunque es muy probable que las cuatro localidades en la vertiente del río Vilcanota presenten la misma composición de especies, es importante mencionar que en la vertiente del río Yanatile, la composición herpetológica difiere (Ochoa, 1996).

Telmatobius marmoratus (Leptodactylidae), se encuentra en la categoría de especie en situación indeterminada, en la lista oficial de especies en peligro para el Perú (Pulido, 1991). 
De los mamíferos reportados $H$. antisiensis se encuentra en situación vulnerable (Pulido, 1991).

Mayor investigación se requiere para conocer la ecología, diversidad y riqueza de la flora y fauna de estos bosques, en orden de tomar decisiones de carácter conservacionista, así también es necesario explorar más esta región para conocer el área remanente de estos bosques, además de ampliar el número de localidades de estudio, e incrementar el número de muestreos y censos, en diferentes estaciones. El presente reporte es una recopilación de la información que se tiene hasta la fecha para los bosques de Polylepis de la Cordillera del Vilcanota, por lo que constituye un punto de partida para el estudio de la flora y fauna en general. En el caso de los mamíferos la lista es incompleta, y se refiere más que todo a mamíferos mayores, aún no se han efectuado muestreos de quirópteros ni roedores, aunque se ha detectado su presencia en dichos bosques. En lo que se refiere a invertebrados, en la actualidad se vienen realizando estudios de diversidad, principalmente de artrópodos, que son el grupo más abundante $\mathrm{y}$ al mismo tiempo menos conocido en éstos bosques.

La falta de información sobre fauna y flora de muchas regiones es una de las mayores dificultades en la elaboración de estrategias de conservación. Los bosques de Polylepis son de origen reciente, sin embargo contienen una fauna y flora distinta que se caracteriza por muchos especialistas de hábitat y altos niveles de endemismo, el cual merece protección. El presente trabajo contribuye con información básica dirigida al conocimiento y preservación de este sistema único de los altos Andes.

\section{Agradecimientos}

Nuestro agradecimiento a los siguientes docentes de la Facultad de Ciencias Biológicas de la UNSAAC: Prof. A.Tupayachi, M. Sc. N. Salinas y Dr. J. L. Venero, asimismo, al Dr. O. Ochoa por compartir información y datos de la colección de anfibios y reptiles. A los egresados y estudiantes: J. Achicahuala, R. Aimituma, I. Aragón, S. Aragón, E. Cárdenas, E. Castro, C. Cevallos, J. Flores, D. Huamán, E. Jarufe, M. Moreno, W. Nauray, M. Olivera, E. Orozco, W. Palomino, B. Quispe, D. Romero, y R. Vera, así como a $\mathrm{M}$. Servat, quienes nos asistieron en el trabajo de campo. En el Herbario de la Universidad Nacional Mayor de San Marcos queremos agradecer a los M. Sc. A. Cano, M. Cano y H. Beltrán por las facilidades otorgadas y su invalorable ayuda en la identificación de especímenes. Asímismo, agradecemos a la Dra. G. Vilcapoma del Departamento de Biología de la Universidad Nacional Agraria de La Molina, por su acertada revisión del artículo. T. Erwin, revisó los primeros manuscritos y realizó valiosos comentarios al texto. El proyecto del cual obtuvimos los datos para el presente estudio, corresponde al proyecto de tesis doctoral del primer autor de este artículo, el cual fue financiado por la National Science Foundation (Dissertation Enhancement Grant \# INT-9724719), American Ornithologist's Union (Carnes Award 1997), Tropical Ecology Fellowship y Parker-Gentry Award, International Center for Tropical Ecology, Univ. of Missouri, St. Louis. Esta es la contribución PQ \# 001 del Proyecto Queñual.

\section{Referencias bibliográficas}

Alegría G. 1995. Estimado poblacional de algunas especies de aves en un bosque natural de Polylepis. Seminario Curricular. Facultad de Ciencias Biológicas. UNSAAC.

Aguilar O. 1998. Evaluación forestal del bosque de Polylepis spp. Mantanay -Yanahuara (Urubamba). Tesis de Biólogo. Facultad de Ciencias Biológicas. UNSAAC.

Aucca C. 1993. Estudio preliminar de la ornitofauna endémica del bosque relicto de Polylepis de Yanacocha (Huayllabamba-Urubamba), Cusco. Medio Ambiente y Desarrollo. Suplemento. :.5-8.

Béjar L. 1996. Flora de los bosques de Polylepis spp. En tres localidades del Valle Sagrado de los Incas. Seminario Curricular. Facultad de Ciencias Biológicas. UNSAAC.

Brako L. y Zarucchi J.L. 1993. Catálogo de las Angiospermas y Gimnospermas del Peru. Monographs in Systematic Botany from the Missouri Botanical Garden. Volume 45.

Collar N.J., Gonzaga L.P., Krabbe N., Matroño N., Naranjo L.J., Parker T.A. III \& Wege D.C. 1992. Threatened birds of the Americas. The ICBP/IUCN Red Data Book. 3rd. ed. Part 2. ICBP, Cambridge, UK.

Ellenberg H. 1958a. Wald oder Steppe? Die naturliche Pflanzendecke der Andes Perus. I Die Umschau. 21: 645-648.

Ellenberg H. 1958b. Wald oder Steppe? Die naturliche Pflanzendecke der Andes Perus. II Die Umschau. 22: 679-681.

Fjeldsa J. 1987. Birds of the relict forests in the high Andes of Peru and Bolivia. Zoological Museum, Copenhagen.

Fjeldsa J. 1992. Biogeographic patterns and evolution of the avifauna of relict high-altitude woodlands of the Andes. Steenstrupia. 18: 9-62.

Fjeldsa J. y Krabbe N. 1990. Birds of the high andes. Zoological Museum. Copenhagen and Apollo books. Svendborg.

Fjeldsa J. \& Kessler M. 1996. Conserving the biological diversity of Polylepis woodlands of the highland of Peru and Bolivia. A contribution to Sustainable Natural Resource Management in the Andes. NORDECO. Copenhagen, Denmark.

Galiano W. 1990. The Flora of Yanacocha; a tropical high andean forest in Southern Peru. Master thesis. 
Department of Biology. University of Missouri at St. Louis.

Hensen I. 1993. Vegetationsokologische Untersuchungen in Polylepis-Waldern der Ostkordillere Boliviens. Diss. Univ. Gottingen.

Holgado T. 1995. El bosque relicto de Polylepis subsericans en quebrada de Pantiacalle, comunidad de Puerto Malaga. Seminario Curricular. Facultad de Ciencias Biológicas. UNSAAC.

Kessler M. 1995. Polylepis Walder Boliviens: Taxa Okologie, Verbreitung und Geschichte. Dissertationes Botanicae 246. J. Cramer. Berlin, Stuttgart.

Koepcke M. 1961. Birds of the western slope of the Andes of Peru. American Museum Novitates. 2028: 1-31.

Ochoa M.O. 1996. Catálogo de vertebrados del Gabinete de Zoología de la UNSAAC. Capítulo III. Anfibios y reptiles. Facultad de Ciencias Biológicas. UNSAAC.

Pulido V. 1991. El libro rojo de la fauna silvestre de Perú. Instituto Nacional de Investigación Agraria y Agroindustrial (INIAA). Lima, Perú.

Simpson B. 1979. A revision of the genus Polylepis (Rosaceae: Sanguisorbeae) Smithsonian Contributions to Botany 43.

Simpson B. 1986. Speciation and specialization of Polylepis in the Andes. Pp 304-315, In Vuilleumier F. y M. Monasterio (eds). High
Altitude Tropical Biogeography. Oxford Univ. Press, New York.

Stotz D. F., Fitzpatrick J.W., Parker T.A.III, \& Moskovitz D.K. 1996. Neotropical Birds: Ecology and Conservation. University of Chicago Press, Ltd., London.

Troll C. 1959. Die tropishe Gebirge. Bonner geographische Abhandlungen. 25:1-93.

Troll C. 1968. The Cordilleras of the Tropical Americas. Coll. Geogr. 9.

Tupayachi A. 1990. Establecimiento de arboretas naturales altoandinos an el Valle Sagrado (CalcaUrubamba) Cusco. Reporte. Facultad de Ciencias Biológicas. UNSAAC.

Venero J.L. y de Macedo H. 1983. Relictos de bosque en la Puna del Perú. Boletín de Lima. 30: 19-26.

Vuilleumier F. 1984. Patchy distribution and systematics of Oreomanes fraseri (Aves: Coerebidae) in Andean Polylepis woodlands. American Museum Novitates. 2777: 1-17.

Walter H. y Medina E. 1969. La temperatura del suelo como determinante para la caracterización de los pisos subalpino y alpino de los Andes de Venezuela. Boletín Venezolano de Ciencias Naturales. 115/116: 201-210.

Weberbauer A. 1945. El mundo vegetal de los Andes peruanos. Lima, Perú: Ministerio de Agricultura.

Wege D.C. \& Long A.J. 1995. Key areas for threatened birds in the neotropics. Birlife Conservation. Series No 5.

Figura 1. Mapa con las localidades de estudio.- 1=Sacsamonte, 2=Yanacocha, 3=Pumahuanca, y 4=Queuñamonte.

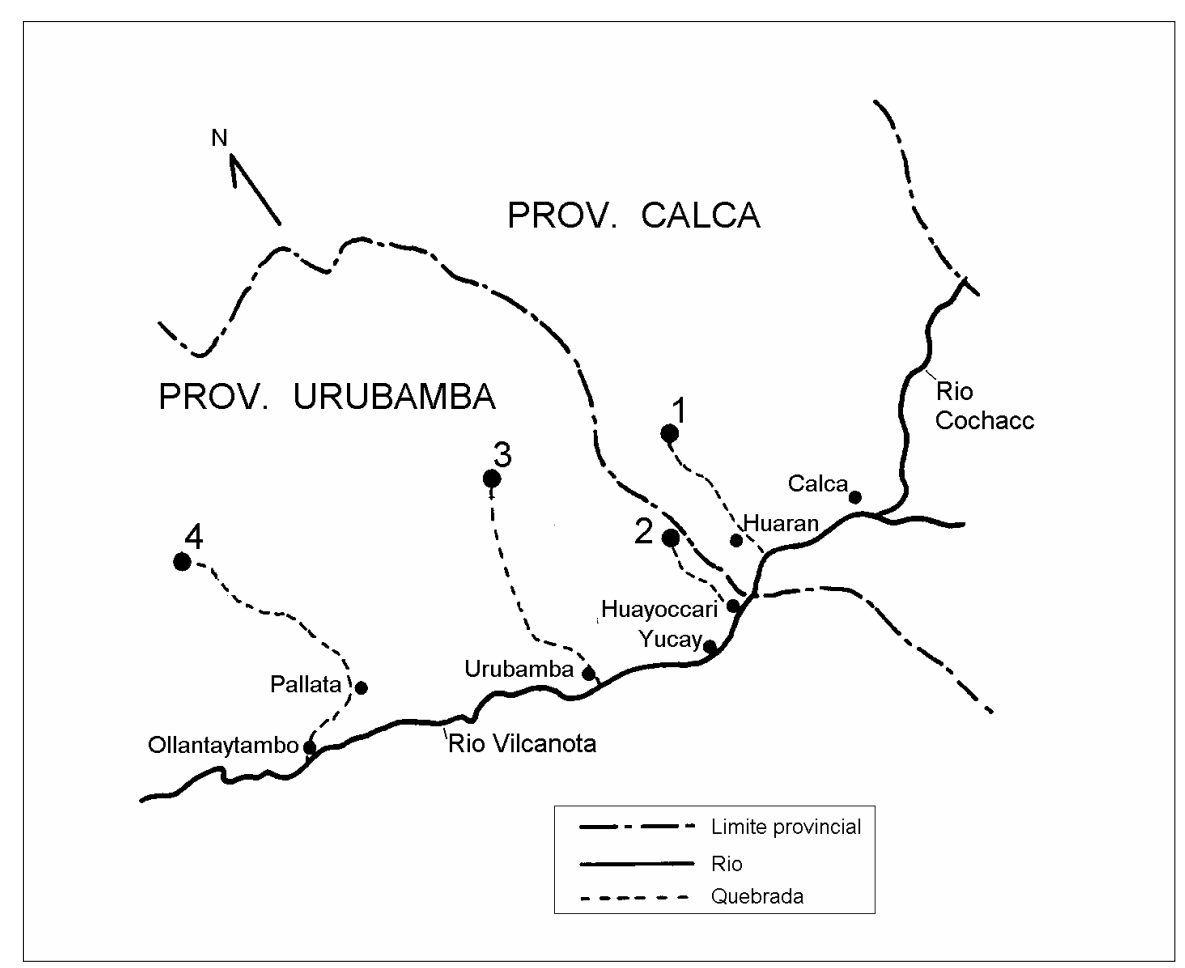


Tabla 1. Listado de plantas Angiospermas asociadas a bosques de Polylepis de la Cordillera del Vilcanota.$\mathrm{S}=$ estación seca, L=estación lluviosa. La información para la estación lluviosa está basada en Béjar (1995) para las localidades de Sacsamonte, Yanacocha y Pumahuanca. Los nombres de las familias y especies sigue la clasificación de Bracko y Zaruchi (1993), salvo excepciones indicadas con un asterisco (*). La letra G indica que la especies ha sido reportada por Galiano (1990) para la localidad de Yanacocha en el bosque de Polylepis propiamente dicho.

\begin{tabular}{|c|c|c|c|c|c|}
\hline Familia & Especie & Sacsamonte & Yanacocha & Pumahuanca & Queuñamonte \\
\hline ALSTROEMERIACEAE* & $\begin{array}{l}\text { Bomarea dulcis (Hooker) } \\
\text { Bomarea aff. Edulis. }\end{array}$ & $\mathrm{SL}$ & $\mathrm{SL}$ & $\begin{array}{l}\mathrm{L} \\
\mathrm{L}\end{array}$ & $\mathrm{S}$ \\
\hline APIACEAE & \begin{tabular}{|l|} 
Azorella multifida $(\mathrm{R}$. \& $\mathrm{P})$. \\
Azorella biloba $($ Schlechtendal) \\
Bowlesia sodiroana H. Wolff \\
Oreomyrrhis andicola (H. B. K.)
\end{tabular} & $\begin{array}{l}\mathrm{L} \\
\mathrm{L}\end{array}$ & $\mathrm{L}$ & $\begin{array}{l}\mathrm{S} \\
\mathrm{L}\end{array}$ & $\mathrm{S}$ \\
\hline ASCLEPIADACEAE & Melinia peruviana (Schlechter) & & $\mathrm{S}$ & & \\
\hline ASTERACEAE & 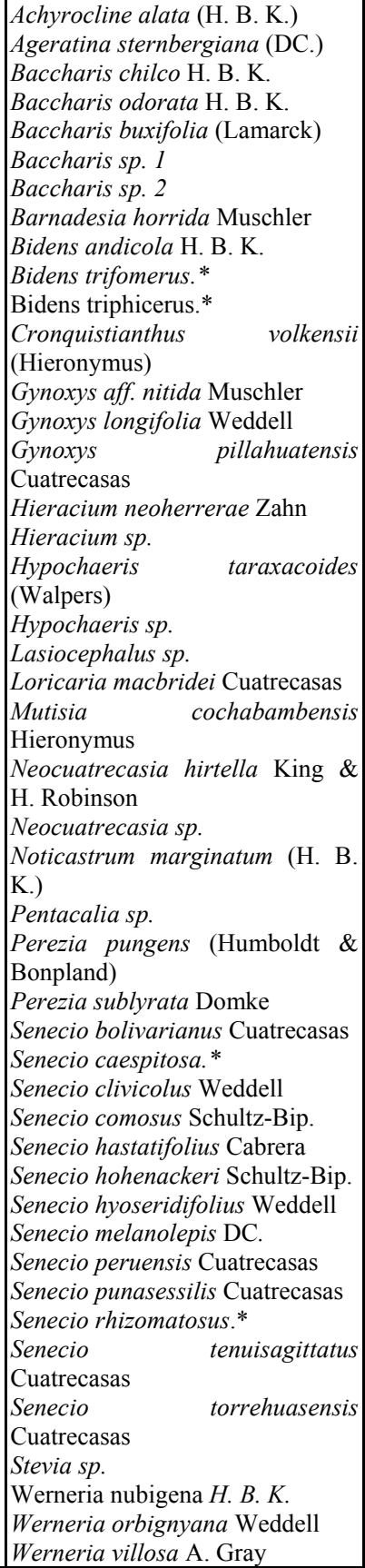 & \begin{tabular}{|l} 
\\
\\
\\
L \\
\\
$\mathrm{L}$ \\
\\
\\
\\
$\mathrm{L}$ \\
$\mathrm{L}$ \\
$\mathrm{SL}$ \\
$\mathrm{S}$ \\
$\mathrm{SL}$ \\
$\mathrm{L}$ \\
$\mathrm{L} \mathrm{SL}$ \\
$\mathrm{S}$ \\
$\mathrm{S}$ \\
$\mathrm{L}$ \\
\\
\end{tabular} & $\begin{array}{l}\mathrm{L} \\
\mathrm{SL} \\
\mathrm{SL} \\
\mathrm{S} \\
\mathrm{S} \\
\mathrm{SL} \\
\mathrm{S}\end{array}$ & \begin{tabular}{|l}
$\mathrm{S}$ \\
$\mathrm{SL}$ \\
$\mathrm{L}$ \\
$\mathrm{SL}$ \\
$\mathrm{S}$ \\
\\
$\mathrm{L}$
\end{tabular} & 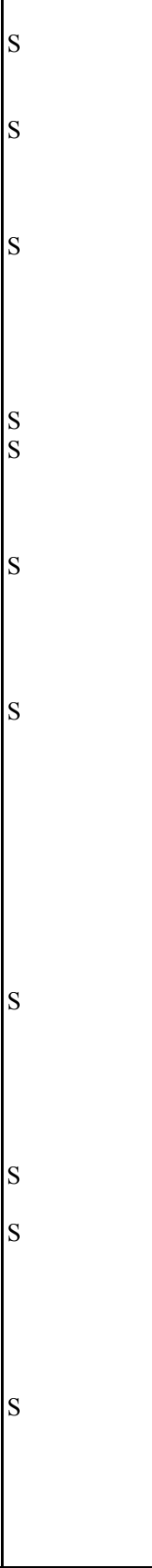 \\
\hline BERBERIDACEAE & Berberis carinata Lechler & $\mathrm{L}$ & SL & $\mathrm{L}$ & \\
\hline
\end{tabular}




\begin{tabular}{|c|c|c|c|c|c|}
\hline & 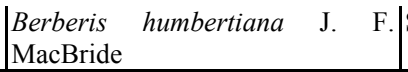 & & $\mathrm{S}$ & S & $\mathrm{S}$ \\
\hline BEGONIACEAE & Begonia veitchii Hooker f. & & G & & \\
\hline BIGNONIACEAE & \begin{tabular}{|l|}
$\begin{array}{l}\text { Eccremocarpus } \\
\text { Sandwith }\end{array}$ \\
\end{tabular} & & $\mathrm{L}$ & & \\
\hline BORAGINACEAE & \begin{tabular}{|l}
$\begin{array}{l}\text { Hackelia } \\
\text { (Schlechtendal \& Chamisso) }\end{array}$ \\
\end{tabular} & & $\mathrm{S}$ & & \\
\hline CARYOPHYLLACEAE & \begin{tabular}{|l|} 
Arenaria lanuginosa (Michaux) \\
Arenaria orbignyana Weddell \\
Arenaria aff. tetragyna. \\
Cerastium vulgatum.* \\
Cerastium glomeratum Thuillier \\
Stellaria cuspidata Wildenow ex \\
Schlechtendal \\
Stellaria media (L.) Cirillo \\
\end{tabular} & $\begin{array}{l}\text { SL } \\
\text { L } \\
\text { L }\end{array}$ & $\mathrm{S}$ & $\begin{array}{l}\mathrm{L} \\
\mathrm{S} \\
\mathrm{L}\end{array}$ & $\mathrm{S}$ \\
\hline CYPERACEAE & \begin{tabular}{|l|} 
Carex pichinchensis H. B. K. \\
\end{tabular} & $\mathrm{L}$ & & & \\
\hline ELAEOCARPACEAE & Vallea stipularis L. f. & & $\mathrm{L}$ & & \\
\hline ERICACEAE & $\begin{array}{|lrr|}\text { Vaccinium } & \text { floribundum } & \text { H. B. K. } \\
\text { Pernettya } & \text { prostrata } & \text { (Cav.) } \\
\text { Sleumer } & & \\
\text { Gaultheria } & \text { glomerata } \\
\text { (Cavanilles) } & & \\
\end{array}$ & S & $\begin{array}{l}\mathrm{L} \\
\mathrm{S}\end{array}$ & $\begin{array}{l}\mathrm{L} \\
\mathrm{S}\end{array}$ & $\mathrm{S}$ \\
\hline FABACEAE & Lupinus aridulus C. P. Smith & & $\mathrm{L}$ & $\mathrm{L}$ & \\
\hline GENTIANACEAE & \begin{tabular}{|l|} 
Gentiana postrata. $^{*}$ \\
Gentianella rima $(\mathrm{D}$. Don ex G. \\
Don) \\
Halenia umbellata $(R . \& P)$. \\
Halenia weddelliana $\mathrm{Gilg}$ \\
\end{tabular} & \begin{tabular}{|l} 
\\
\\
SL \\
SL
\end{tabular} & $\mathrm{SL}$ & $\begin{array}{l}\mathrm{L} \\
\mathrm{L} \\
\mathrm{L} \\
\mathrm{SL} \\
\end{array}$ & $\begin{array}{l}\mathrm{S} \\
\mathrm{S}\end{array}$ \\
\hline GERANIACEAE & \begin{tabular}{|l|} 
Geranium diffusum $\mathrm{H} . \mathrm{B} . \mathrm{K}$. \\
Geranium filipes \\
Gillip \\
Geranium herrerae \\
Gnuth \\
Heranium peronymus
\end{tabular} & \begin{tabular}{|l}
$\mathrm{L}$ \\
$\mathrm{L}$ \\
$\mathrm{L}$ \\
\end{tabular} & $\begin{array}{l}\mathrm{L} \\
\mathrm{S}\end{array}$ & $\mathrm{L}$ & \\
\hline GROSSULARIACEAE & \begin{tabular}{|l|} 
Escallonia myrtilloides (R. \& P.) \\
Ribes brachybotrys (Weddell)
\end{tabular} & $\mathrm{SL}$ & $\begin{array}{l}\mathrm{S} \\
\mathrm{SL} \\
\end{array}$ & SL & $\begin{array}{l}\mathrm{S} \\
\mathrm{S} \\
\end{array}$ \\
\hline IRIDACEAE & Sisyrinchium junceum.* & & $\mathrm{L}$ & & \\
\hline JUNCACEAE & \begin{tabular}{|l|} 
Luzula gigantea Desvaux \\
Luzula racemosa Desvaux \\
Luzula peruviana.*
\end{tabular} & $\begin{array}{l}\mathrm{L} \\
\mathrm{L} \\
\mathrm{S} \\
\end{array}$ & $\mathrm{L}$ & $\mathrm{L}$ & \\
\hline LAMIACEAE & \begin{tabular}{|l|}
$\begin{array}{l}\text { Satureja boliviana } \text { (Bentham) } \\
\text { Stachys herrerae Epling }\end{array}$ \\
\end{tabular} & SL & $\mathrm{S}$ & \begin{tabular}{|l}
$\mathrm{SL}$ \\
$\mathrm{L}$
\end{tabular} & \\
\hline LOASACEAE & $\begin{array}{|ll|}\begin{array}{l}\text { Caiophora } \\
\text { (Desrousseaux) }\end{array} & \text { contorta } \\
\end{array}$ & SL & SL & $\mathrm{SL}$ & $\mathrm{S}$ \\
\hline MELASTOMATACEAE & Brachyotum naudinii Triana & & $\mathrm{S}$ & SL & $\mathrm{S}$ \\
\hline ONAGRACEAE & Fuchsia apetala R. \& P. & $\mathrm{S}$ & SL & SL & $\mathrm{S}$ \\
\hline ORCHIDACEAE & Aa sp. & & & $\mathrm{S}$ & \\
\hline OXALIDACEAE & $\begin{array}{l}\text { Oxalis aff. petrophila Knuth } \\
\text { Oxalis lotoides H. B. K. }\end{array}$ & $\begin{array}{l}\mathrm{L} \\
\mathrm{S}\end{array}$ & SL & $\mathrm{S}$ & $\mathrm{S}$ \\
\hline PASSIFLORACEAE & Passiflora trifoliata Cavanilles & & & $\mathrm{S}$ & \\
\hline PIPERACEAE & Peperomia sp. & & $\mathrm{L}$ & & \\
\hline PLANTAGINACEAE & Plantago lamprophylla Pilger & & $\mathrm{G}$ & & \\
\hline POACEAE & 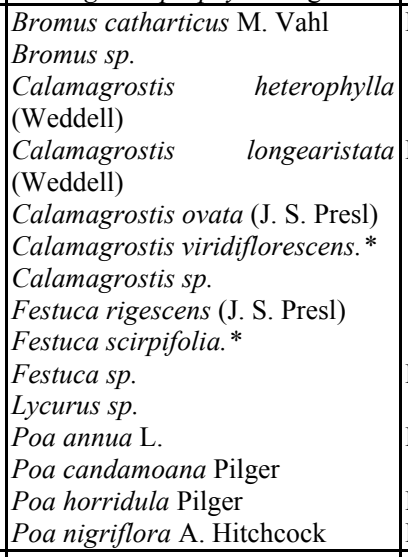 & $\begin{array}{l}\mathrm{L} \\
\mathrm{L} \\
\\
\mathrm{L} \\
\mathrm{L} \\
\mathrm{L} \\
\mathrm{L} \\
\end{array}$ & $\begin{array}{l}\mathrm{L} \\
\mathrm{L} \\
\mathrm{L}\end{array}$ & \begin{tabular}{|l}
$\mathrm{L}$ \\
$\mathrm{L}$ \\
$\mathrm{L}$ \\
$\mathrm{L}$ \\
$\mathrm{L}$ \\
$\mathrm{L}$ \\
$\mathrm{L}$ \\
$\mathrm{L}$ \\
$\mathrm{L}$ \\
$\mathrm{L}$ \\
$\mathrm{L}$ \\
$\mathrm{L}$ \\
$\mathrm{L}$ \\
$\mathrm{L}$
\end{tabular} & \\
\hline POLYGALACEAE & Monnina salicifolia R. \& P. & & $\mathrm{S}$ & $\mathrm{SL}$ & $\mathrm{S}$ \\
\hline POLYGONACEAE & Muehlenbeckia volcanica & & $\mathrm{S}$ & $\mathrm{SL}$ & $\mathrm{S}$ \\
\hline
\end{tabular}


Ecología Aplicada Vol. 1, No 1, pp. 25 - 35

\begin{tabular}{|c|c|c|c|c|c|}
\hline & $\mid \begin{array}{l}\text { (Bentham) } \\
\text { Rumex acetosella } \mathrm{L} .\end{array}$ & $\mathrm{s}$ & $\mathrm{L}$ & & \\
\hline RANUNCULACEAE & \begin{tabular}{|l} 
Ranunculus cymbalaria Pursh \\
Ranunculus praemorsus H. B. K. \\
Krapfia macropetala.*
\end{tabular} & $\begin{array}{l}\mathrm{L} \\
\mathrm{L} \\
\mathrm{S}\end{array}$ & $\begin{array}{l}\mathrm{L} \\
\text { S }\end{array}$ & $\mathrm{L}$ & \\
\hline ROSACEAE & 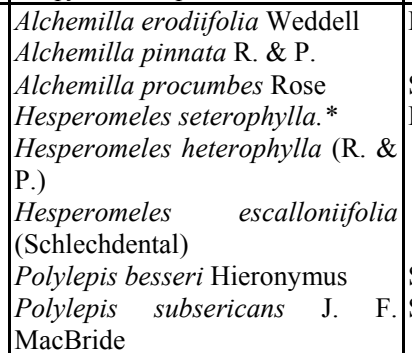 & $\begin{array}{l}\mathrm{L} \\
\mathrm{S} \\
\mathrm{L} \\
\mathrm{L} \\
\\
\\
\mathrm{SL} \\
\mathrm{SL}\end{array}$ & $\begin{array}{l}\text { SL } \\
\text { SL }\end{array}$ & $\begin{array}{l}\text { SL } \\
\text { SL }\end{array}$ & \\
\hline RUBIACEAE & \begin{tabular}{|l|} 
Galium hypocarpium (L.) \\
Galium sp. 1
\end{tabular} & & $\overline{\mathrm{S}}$ & $\overline{\mathrm{S}}$ & \\
\hline SCROPHULARIACEAE & \begin{tabular}{|l|} 
Bartsia bartsioides (Hooker) \\
Bartsia diffusa Bentham \\
Calceolaria engleriana. \\
Calceolaria virgata R. \& P. \\
Castilleja fissifolia L. f. \\
Ourisia chamaedryfolia Bentham \\
Veronica serpyllifolia L.
\end{tabular} & $\begin{array}{l}\mathrm{S} \\
\mathrm{L} \\
\mathrm{SL} \\
\mathrm{S}\end{array}$ & $\begin{array}{l}\text { SL } \\
\text { L } \\
\text { L }\end{array}$ & $\begin{array}{l}\text { S } \\
\text { SL } \\
\text { S }\end{array}$ & \\
\hline SOLANACEAE & \begin{tabular}{|l|} 
Salpichroa glandulosa (Hooker) \\
Salpichroa micrantha Benoist \\
Salpichroa hirsuta (Meyen) \\
Solanum acaule Bitter \\
Solanum raphanifolium Cardenas \\
and Hawkes \\
Solanum sp.
\end{tabular} & $\begin{array}{l}\mathrm{L} \\
\mathrm{L} \\
\mathrm{L} \\
\mathrm{L} \\
\mathrm{L}\end{array}$ & $\mathrm{G}^{*}$ & S & \\
\hline URTICACEAE & \begin{tabular}{|l|} 
Urtica echinata Bentham \\
\end{tabular} & SL & $\mathrm{SL}$ & $\mathrm{SL}$ & \\
\hline VALERIANACEAE & \begin{tabular}{|l|} 
Valeriana decussata $\mathrm{R} . \&$ P. \\
Valeriana micropterina Weddell \\
Valeriana nivalis Weddell \\
\end{tabular} & $\begin{array}{l}\mathrm{L} \\
\mathrm{S}\end{array}$ & $\begin{array}{l}\mathrm{S} \\
\mathrm{SL}\end{array}$ & SL & \\
\hline VERBENACEAE & \begin{tabular}{|l|}
$\begin{array}{l}\text { Citharexylum argutedentatum } \\
\text { Moldenke }\end{array}$ \\
\end{tabular} & & $\bar{S}$ & & \\
\hline
\end{tabular}


Tabla 2. Listado de aves asociadas a bosques de Polylepis de la Cordillera del Vilcanota.-1. Abundancia: $\mathrm{C}=$ común; más que 10 individuos de una especie son encontrados en el hábitat apropiado diariamente. $\mathrm{U}=$ poco común; individuos de una misma especie en el hábitat apropiado, son encontrados menos del 50\% del tiempo durante la estación de campo. $\mathrm{R}=$ raro; uno o muy pocos encuentros durante la estación de campo. 2. Hábitat: $\mathrm{A}=$ aéreo, se refiere al espacio en el cual las aves pueden ser observadas en vuelo sobre otros hábitats. $\mathrm{C}=$ claro de bosque (muchos de estos corresponden a roquedales, bofedales, y/o pastizales). AR=vegetación arbustiva. $\mathrm{BB}=$ borde del bosque. $\mathrm{BO}=$ bofedal. $\mathrm{BP}=$ bosque de Polylepis propiamente dicho. $\mathrm{L}=$ lagunas. $\mathrm{OL}=$ orilla de lagunas. $\mathrm{PA}=$ pastizal. $\mathrm{Q}=$ quebradas. $\mathrm{R}=$ =roquerío. 3. Estrato: $\mathrm{A}=$ aéreo. $\mathrm{Ag}=$ sobre el agua. $\mathrm{T}=$ terrestre (se le encuentra mayormente en el suelo). $\mathrm{S}=$ sotobosque, nivel de vegetación entre los $0-4 \mathrm{~m}$. $\mathrm{SD}=$ subdosel, nivel comprendido entre los $4-8 \mathrm{~m}$ de altura. $\mathrm{D}=$ dosel, nivel de vegetación donde la copa de los árboles forma una capa contínua (alcanza $14 \mathrm{~m}$ en algunos bosques). Las negritas indican las especies del bosque propiamente dicho. El asterisco posterior al nombre indica que se encuentra listada como especie amenazada.

\begin{tabular}{|c|c|c|c|c|c|c|c|}
\hline \multirow[b]{2}{*}{ Familia } & \multirow[b]{2}{*}{ Especies } & \multicolumn{4}{|c|}{ ABUNDANCIA } & \multirow[t]{2}{*}{ HABITAT } & \multirow[t]{2}{*}{ ESTRATO } \\
\hline & & Sacsamonte & Yanacocha & Pumahuanca & Queuñamonte & & \\
\hline PODICIPEDIDAE & Podiceps occipitalis & & C & & & $\mathrm{L}$ & $\mathrm{Ag}$ \\
\hline CATHARTIDAE & Vultur gryphus & $\mathrm{R}$ & & & & $\mathrm{A}$ & $\mathrm{A}$ \\
\hline ACCIPITRIDAE & \begin{tabular}{|l|} 
Circus cinereus \\
Geranoaetus \\
melanoleucus \\
Buteo polyosoma \\
Buteo poecilochrous \\
\end{tabular} & $\mathrm{U}$ & $\begin{array}{l}\mathrm{R} \\
\mathrm{U}\end{array}$ & $\mathrm{U}$ & $\mathrm{U}$ & $\begin{array}{l}\mathrm{A} \\
\mathrm{A} \\
\mathrm{A} \\
\mathrm{A}\end{array}$ & $\begin{array}{l}\mathrm{A} \\
\mathrm{A} \\
\mathrm{A} \\
\mathrm{A}\end{array}$ \\
\hline FALCONIDAE & $\begin{array}{l}\text { Phalcoboenus } \\
\text { megalopterus } \\
\text { Falco sparverius }\end{array}$ & C & $\mathrm{C}$ & C & C & A & $\begin{array}{l}\mathrm{T}, \mathrm{S}, \mathrm{SD}, \mathrm{D} \\
\mathrm{A}\end{array}$ \\
\hline $\begin{array}{l}\text { ANATIDAE } \\
\text { SCOLOPACIDAE }\end{array}$ & \begin{tabular}{|l} 
Chloephaga \\
melanoptera \\
Gallinago andina
\end{tabular} & C & $U$ & $\mathrm{U}$ & & $\begin{array}{l}\text { OL, BO } \\
\text { OL, BO }\end{array}$ & $\begin{array}{l}\mathrm{T} \\
\mathrm{T}\end{array}$ \\
\hline LARIDAE & Larus serranus & & $\mathrm{C}$ & & & $\mathrm{OL}, \mathrm{BO}, \mathrm{L}$ & $\mathrm{T}, \mathrm{A}, \mathrm{Ag}$ \\
\hline COLUMBIDAE & $\begin{array}{l}\text { Columba maculosa } \\
\text { Metriopelia } \\
\text { melanoptera }\end{array}$ & & $\begin{array}{l}\mathrm{U} \\
\mathrm{U}\end{array}$ & & & $\begin{array}{l}\mathrm{C} \\
\mathrm{BB}, \mathrm{BP}\end{array}$ & $\begin{array}{l}\mathrm{A} \\
\mathrm{SD}\end{array}$ \\
\hline PSITTACIDAE & \begin{tabular}{|l|}
$\begin{array}{l}\text { Bolborhynchus } \\
\text { orbygnesius }\end{array}$ \\
\end{tabular} & & $\mathrm{U}$ & & & $\mathrm{A}, \mathrm{BP}$ & D \\
\hline STRIGIDAE & \begin{tabular}{|l|} 
Bubo virginianus \\
Glaucidium jardinii
\end{tabular} & & $\begin{array}{l}\mathrm{U} \\
\mathrm{U}\end{array}$ & & & $\begin{array}{l}\mathrm{R}, \mathrm{BB} \\
\mathrm{Q}\end{array}$ & \begin{tabular}{|l} 
SD \\
SD \\
\end{tabular} \\
\hline CAPRIMULGIDAE & \begin{tabular}{|l|} 
Caprimulgus \\
longirostris \\
\end{tabular} & & $\mathrm{U}$ & & & $\mathrm{BB}$ & $\mathrm{T}, \mathrm{S}$ \\
\hline TROCHILIDAE & \begin{tabular}{|l} 
Patagona gigas \\
Colibri coruscans \\
Oreotrochilus estella \\
Aglaeactis \\
castelnaudii \\
Lesbia nuna \\
Metallura \\
tyrianthina \\
Chalcostigma \\
stanleyi \\
\end{tabular} & C & $\begin{array}{l}\mathrm{U} \\
\mathrm{U} \\
\mathrm{R} \\
\mathrm{C} \\
\mathrm{R} \\
\mathrm{R} \\
\mathrm{C} \\
\mathrm{C}\end{array}$ & C & $\begin{array}{l}\mathrm{R} \\
\mathrm{C} \\
\mathrm{C} \\
\mathrm{C}\end{array}$ & \begin{tabular}{|l}
$\mathrm{BB}$ \\
$\mathrm{BB}$ \\
$\mathrm{BP}, \mathrm{C}$ \\
$\mathrm{BP}$ \\
$\mathrm{BP}$ \\
$\mathrm{BP}$ \\
$\mathrm{BP}$
\end{tabular} & \begin{tabular}{|l} 
SD, D \\
S, SD, D \\
S, SD \\
S, SD, D \\
S, SD, D \\
S, SD, D \\
S, SD, D
\end{tabular} \\
\hline PICIDAE & $\begin{array}{l}\text { Veniliornis nigriceps } \\
\text { Colaptes rupicola }\end{array}$ & $\begin{array}{l}\mathrm{U} \\
\mathrm{U}\end{array}$ & $\begin{array}{l}\mathrm{U} \\
\mathrm{U}\end{array}$ & $\mathrm{U}$ & $\mathrm{U}$ & \begin{tabular}{|l|} 
BP \\
C
\end{tabular} & \begin{tabular}{|l} 
SD, D \\
$T$
\end{tabular} \\
\hline FURNARIIDAE & \begin{tabular}{|l|} 
Upucerthia jelskii \\
Cinclodes fuscus \\
Cinclodes excelsior* \\
Leptasthenura \\
yanacensis* \\
Leptasthenura \\
xenothora** \\
Cranioleuca \\
albicapilla* \\
Asthenes \\
urubambensis* \\
Asthenes ottonis \\
\end{tabular} & $\begin{array}{l}\text { C } \\
\text { U } \\
\text { U } \\
\text { C } \\
\text { C } \\
\text { R } \\
\text { U }\end{array}$ & $\begin{array}{l}\mathrm{C} \\
\mathrm{U}\end{array}$ & \begin{tabular}{|l}
$\mathrm{R}$ \\
$\mathrm{C}$ \\
$\mathrm{U}$ \\
$\mathrm{U}$ \\
$\mathrm{C}$ \\
$\mathrm{C}$
\end{tabular} & C & $\begin{array}{l}\mathrm{PA} \\
\mathrm{Q}, \mathrm{BO} \\
\mathrm{BP} \\
\mathrm{BP} \\
\mathrm{BP} \\
\mathrm{BP} \\
\mathrm{BB}, \mathrm{BP} \\
\mathrm{BB}, \mathrm{BP} \\
\end{array}$ & $\begin{array}{l}\text { T } \\
\text { T } \\
\text { T } \\
\text { S, SD, D } \\
\text { S, SD, D } \\
\text { S, SD, D } \\
\text { T, S } \\
\text { T, S } \\
\end{array}$ \\
\hline FORMICARIIDAE & Grallaria andicola $*$ & $\mathrm{U}$ & $\mathrm{C}$ & $\mathrm{C}$ & $\mathrm{C}$ & BP & $\mathrm{T}, \mathrm{S}$ \\
\hline RHINOCRIPTIDAE & $\begin{array}{l}\text { Scytalopus } \\
\text { magellanicus }\end{array}$ & C & C & C & C & $\mathrm{BP}$ & $\mathrm{T}$ \\
\hline TYRANNIDAE & \begin{tabular}{|l|} 
Mecocerculus \\
leucophrys \\
Anairetes alpinus*
\end{tabular} & $\mathrm{R}$ & $\mathrm{U}$ & U & R & $\begin{array}{l}\mathrm{BP} \\
\mathrm{BP}\end{array}$ & $\begin{array}{l}\text { SD, D } \\
\text { SD, D }\end{array}$ \\
\hline
\end{tabular}


Ecología Aplicada Vol. 1, No 1, pp. $25-35$

\begin{tabular}{|c|c|c|c|c|c|c|c|}
\hline & $\mid$\begin{tabular}{|l|} 
Anairetes parulus \\
Octhoeca \\
rufipectoralis \\
Octhoeca leucophrys \\
Octhoeca \\
oenanthoides* \\
Cnemarcus \\
erythropygius \\
Polioxolmis \\
rufipennis* \\
Muscisaxicola \\
alpina \\
Muscisaxicola \\
rufivertex
\end{tabular} & $\begin{array}{l}\mathrm{U} \\
\mathrm{U} \\
\mathrm{U} \\
\mathrm{U} \\
\mathrm{R} \\
\mathrm{U}\end{array}$ & $\mid \begin{array}{l}\mathrm{U} \\
\mathrm{C} \\
\mathrm{U} \\
\mathrm{U}\end{array}$ & $\mid \begin{array}{l}\mathrm{U} \\
\mathrm{C}\end{array}$ & $\begin{array}{l}\mathrm{U} \\
\mathrm{C}\end{array}$ & $\begin{array}{l}\mathrm{BP}, \mathrm{AR} \\
\mathrm{BB}, \mathrm{BP} \\
\mathrm{BP}, \quad \mathrm{AR}, \\
\mathrm{OL} \\
\mathrm{BP} \\
\mathrm{BB} \\
\mathrm{BB}, \mathrm{PA} \\
\mathrm{PA}, \mathrm{BO} \\
\mathrm{PA}, \mathrm{BO}\end{array}$ & $\begin{array}{l}\text { SD, D } \\
\text { SD, D } \\
\text { T, S } \\
\text { S } \\
\text { U, SD, D } \\
\text { T, S } \\
\text { T } \\
\text { T }\end{array}$ \\
\hline HIRUNDINIDAE & \begin{tabular}{|l} 
Notiochelidon \\
murina \\
Petrochelidon \\
andecola
\end{tabular} & C & C & $\mathrm{C}$ & C & A & A \\
\hline TROGLODYTIDAE & Troglodytes aedon & C & C & C & $\mathrm{C}$ & BP & $\mathrm{S}$ \\
\hline CINCLIDAE & \begin{tabular}{|l|}
$\begin{array}{l}\text { Cinclus } \\
\text { leucocephalus }\end{array}$ \\
\end{tabular} & $\mathrm{U}$ & & $\mathrm{U}$ & $\mathrm{U}$ & $\mathrm{Q}$ & $\mathrm{T}$ \\
\hline TURDIDAE & \begin{tabular}{|l|} 
Turdus fuscater \\
Turdus chiguanco
\end{tabular} & C & $\begin{array}{l}\mathrm{C} \\
\mathrm{C} \\
\end{array}$ & $\begin{array}{l}\mathrm{C} \\
\mathrm{C} \\
\end{array}$ & C & \begin{tabular}{|l}
$\mathrm{BP}$ \\
$\mathrm{BP}$
\end{tabular} & $\begin{array}{l}\text { S, SD } \\
\text { S, SD }\end{array}$ \\
\hline COEREBIDAE & \begin{tabular}{|l|} 
Conirostrum \\
cinereum \\
Conirostrum \\
ferrugineiventre \\
Oreomanes fraseri* \\
Xenodacnis parina* \\
Diglossa \\
brunneiventris \\
\end{tabular} & $\begin{array}{l}\text { C } \\
\text { C } \\
\text { C } \\
\text { U } \\
\end{array}$ & $\begin{array}{l}\mathrm{C} \\
\mathrm{C} \\
\mathrm{U}\end{array}$ & $\begin{array}{l}\mathrm{C} \\
\mathrm{C} \\
\mathrm{C}\end{array}$ & $\begin{array}{l}\text { C } \\
\text { C }\end{array}$ & $\begin{array}{l}\mathrm{BP} \\
\mathrm{BP} \\
\mathrm{BP} \\
\mathrm{BP} \\
\mathrm{BB}, \mathrm{BP}\end{array}$ & $\begin{array}{l}\mathrm{S} \\
\mathrm{S} \\
\mathrm{S}, \mathrm{SD} \\
\text { S, SD } \\
\text { S, SD }\end{array}$ \\
\hline THRAUPIDAE & \begin{tabular}{|l|l|}
$\begin{array}{l}\text { Thraupis } \\
\text { bonariensis }\end{array}$ \\
\end{tabular} & $\mathrm{U}$ & $\mathrm{U}$ & $\mathrm{U}$ & & $\mathrm{BP}, \mathrm{PA}$ & $\mathrm{T}, \mathrm{S}, \mathrm{SD}$ \\
\hline FRINGILLIDAE & \begin{tabular}{|} 
Catamenia inornata \\
Diuca speculifera \\
Phrygilus punensis \\
Phrygilus fruticeti \\
Phrygilus unicolor \\
Phrygilus plebejus \\
Zonotrichia capensis \\
Carduelis \\
rrassirostris* \\
Carduelis \\
magellanica \\
Carduelis atrata
\end{tabular} & $\begin{array}{l}\text { C } \\
\text { C } \\
\text { C } \\
\text { C } \\
\text { C } \\
\text { U } \\
\\
\text { C }\end{array}$ & $\begin{array}{l}\mathrm{C} \\
\mathrm{U} \\
\mathrm{C} \\
\mathrm{C} \\
\mathrm{C} \\
\mathrm{U}\end{array}$ & $\begin{array}{l}\mathrm{C} \\
\mathrm{U}\end{array}$ & $\mid \begin{array}{l}\mathrm{C} \\
\mathrm{R} \\
\mathrm{C}\end{array}$ & $\begin{array}{l}\text { BB, AR } \\
\text { BO } \\
\text { BB, C, AR } \\
\text { BB, C, AR } \\
\text { BB, C, AR } \\
\text { BB, C, AR } \\
\text { BB, C, AR } \\
\text { BP } \\
\text { BB, AR } \\
\text { BB }\end{array}$ & $\begin{array}{l}\text { S } \\
\text { T } \\
\text { T, S } \\
\text { T, S } \\
\text { T, S } \\
\text { T, S } \\
\text { T, S } \\
\text { T, SD, D } \\
\text { SD, D } \\
\text { SD, D }\end{array}$ \\
\hline
\end{tabular}

Tabla 3. Listado de anfibios y reptiles asociados a bosques de Polylepis de la Cordillera del Vilcanota.Abundancia: $\mathrm{C}=$ común, $\mathrm{U}=$ poco común, $\mathrm{R}=$ raro. El asterisco indica que la especie está asociada con cuerpos de agua por lo que no es posible asignarle un rango de distribución.

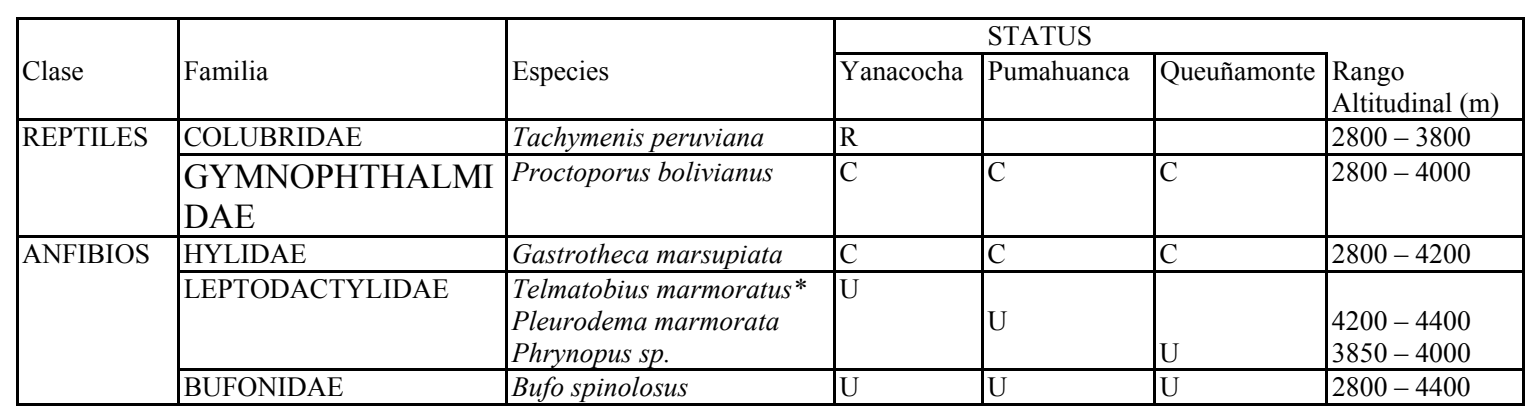

